\title{
Finite-dimensional Vector Spaces with Frobenius Action
}

\author{
Florian Enescu
}

\begin{abstract}
We present the structure of finite-dimensional vector spaces that admit a Frobenius action, with emphasis on the case of vector spaces over a perfect field where the module theory over noncommutative principal ideal domains allows for a unified treatment.
\end{abstract}

Keywords. Frobenius Homomorphism, Frobenius Action, Antinilpotent Module, Noncommutative PID, Perfect Field.

2010 Mathematics Subject Classification. 13A35.

\section{Introduction}

Among all the modules defined over a commutative ring of positive characteristic a special class arises naturally. These are the modules that admit a Frobenius action and their properties have been studied extensively in commutative algebra in recent years. More precisely, if $A$ is a commutative ring of prime characteristic $p$, then, for an $A$ module $M$, a Frobenius action is an additive map $F_{M}: M \rightarrow M$ such that $F_{M}(\mathrm{am})=$ $a^{p} F_{M}(m)$ for all $a \in A, m \in M$. Perhaps the most important example of nontrivial $A$-modules with a natural Frobenius action is given by the local cohomology modules of $A$ with support in an ideal $I$ of $A$, namely $H_{I}^{i}(A), i=0,1, \ldots$ The Frobenius action on these local cohomology modules is induced from the natural action on the ring via the Čech complex. One remarkable point is that important properties of a local ring $(A, \mathfrak{m})$ can be often rephrased in terms of the Frobenius action on the local cohomology modules of $A$ with support in its maximal ideal. This approach has been developed and exploited by many of authors who produced significant results in the theory of rings of positive characteristic, with many of these results connected to either tight closure theory or birational geometry (see [4-9, 11-13, 17, 18, 20-25, 30-41] for a representative but incomplete list).

The particular case of finite dimensional vector spaces that admit a Frobenius action has connections to other areas of mathematics, such as number theory and Lie algebras. However, this case was not systematically studied and presented, with results developed only as needed, scattered through the literature, and often rediscovered by different authors. A coherent and complete presentation of this theory has not been available so far, even if it is considered a classical subject. Work for the vector space

The author was partially supported by the NSA Young Investigator Grant H98230-10-1-0166. 
case has been included in papers of Blickle and Böckle [9], Hochster [17], Harthsorne and Speiser [16], Lyubeznik [23] and Yoshino [43]. This paper is meant to serve the commutative algebra community, by providing a place where the contributions on this subject are collected. Moreover, there is one particular current direction of research where we expect that the study here will be useful. The notion of F-injectivity for rings of positive characteristic has been studied at length in the past decade or so. Most of the work has been carried in the case of Cohen-Macaulay rings where F-injectivity is considerably easier to handle. We expect that the results and techniques collected here to help in the study of F-injective rings that are generalized Cohen-Macaulay. In this case, the local cohomology modules of index strictly less that the dimension of the ring have finite length, and hence they could be investigated with the tools presented here. We included one result, Theorem 7.14, that illustrates this idea.

Our plan is to provide a unifying point of view to the theory of finite dimensional vector spaces with a Frobenius action, including the most important results of the theory and exploring connections to current research. We framed our presentation by following some classical work of N. Jacobson which applies naturally in the case of vector spaces over a perfect field, and puts the theory in a new light. Moreover, it gives the opportunity to the reader to rediscover and appreciate important work in module theory which nowadays is not included in standard textbooks of modern algebra. This work is the classification of finitely generated modules over noncommutative principal ideal domains based on the work of Asano [1,2], Nakayama [29], Teichmüller [42], and presented beautifully by Jacobson in his classical treatise [26].

\section{A Noncommutative Principal Ideal Domain}

We will first introduce the objects of our study. Let $K$ be a field of positive characteristic $p$, where $p$ is prime. Then $K$ admits the natural Frobenius homomorphism defined by $F(k)=k^{p}$, for all $k \in K$.

Let $K\{F\}$ be the skew polynomial ring in the indeterminate $F$ with coefficients in $K$, modulo the relations given by $F k=k^{p} F$, for all $k \in K$.

More precisely, this ring is defined by considering the set of all formal expressions $\sum_{i=0}^{n} a_{i} F^{i}$ where $F$ is an indeterminate, $n \in \mathbb{N}$ and $a_{i} \in K$ for all $i=0, \ldots, n$. The elements of this set will be called polynomials in $F$ by analogy to the commutative case. Endow this set with the operations of addition and multiplication in the following way: the addition is the natural addition operation of polynomials and the multiplication has the property that

$$
a F^{k} \cdot b F^{l}:=a F^{k} b F^{l}=a b^{p^{k}} F^{k} F^{l}=a b^{p^{k}} F^{k+l},
$$

for all $a, b \in K, k, l \in \mathbb{N}$, and it is extended to arbitrary polynomials via the distributive property of multiplication over addition. 
It is a routine check to verify that this set together with these two operations forms a noncommutative ring. We denote this ring by $K\{F\}$ and call its elements (skew) polynomials.

This construction can be performed in a more general setting where $K$ can be replaced by any ring $R$ and $F$ by a ring endomorphism of $R$. Rings obtained in such way are called skew polynomials rings and are a basic construction in noncommutative ring theory, see Chaper 1 in [15] and Chapter 1 in [28].

Let $V$ be a finite dimensional $K$-vector space and $F_{V}: V \rightarrow V$ a Frobenius action on $V$, that is $F_{V}$ is additive and $F_{V}(k v)=k^{p} F_{V}(v)$ for all $k \in K$ and $v \in V$. Allowing a Frobenius action on $V$ is equivalent to endowing $V$ with a left $K\{F\}$ module structure by putting $F \cdot v=F_{V}(v)$. For these reasons, we will simply denote the action on $V$ by $F=F_{V}$.

Therefore the study of vector spaces with a Frobenius action is equivalent to that of the category of left modules over the skew polynomial ring $K\{F\}$. Skew polynomial rings have been long-studied, and in what follows we will present the applications to their module theory with an eye towards the case we have in mind, that of finite dimensional vector spaces with a Frobenius action. It should be noted here that a notion of Cartier modules has been defined recently by Blickle and Böckle in [9]. A vector space $V$ over a field $K$ of positive characteristic $p$ is called a Cartier vector space if it admits a Cartier map, that is an additive map $C_{V}: V \rightarrow V$ such that $C_{V}\left(a^{p} v\right)=a C_{V}(v)$ for all $a \in K, v \in V$. These vector spaces are exactly the right modules over the ring $K\{F\}$. The connection between left and right modules over the skew polynomial ring have been studied recently in [36] in addition to [9]. The theory that we present in this paper can be developed analogously for right modules, but we will not pursue it here.

If $f=a_{0}+a_{1} F+\cdots+a_{n} F^{n} \in K\{F\}$ with $a_{n} \neq 0$, we say that $f$ has degree $n$ and write $\operatorname{deg}(f)=n$. Using the degree function one can easily check that $K\{F\}$ is a domain. The degree function also gives a division and remainder type theorem:

Proposition 2.1. Let $f, g$ be two polynomials in $K\{F\}$. Assume that $g \neq 0$.

(i) There exists uniquely determined $q, r \in K\{F\}$ such that $f=q g+r$ and either $r=0$ or $\operatorname{deg}(r)<\operatorname{deg}(g)$.

(ii) Assume that $F: K \rightarrow K$ is a automorphism, i.e. $K$ is perfect. Then there exists uniquely determined $q, r \in K\{F\}$ such that $f=g q+r$ and either $r=0$ or $\operatorname{deg}(r)<\operatorname{deg}(g)$.

Proof. The proof is a routine exercise.

This proposition enables one to show the following important result (see Theorem 1.11 in [15] for a more general setting and a proof). 
Theorem 2.2. The ring $K\{F\}$ is a left principal ideal domain (i.e. all left ideals are principal). If $K$ is perfect then $K\{F\}$ is a left and right principal ideal domain (i.e. all left ideals and all right ideals are principal).

Definition 2.3. A noncommutative ring $R$ that is a left and right principal ideal domain will be called a noncommutative PID. In this paper, all such examples will be of noncommutative rings, so a noncommutative PID will be sometimes called simply a PID.

We have the following important properties of a noncommutative PID.

Proposition 2.4. Let $R$ be a noncommutative PID. Then $R$ is left and right Noetherian. Moreover, the descending chain condition holds for chains of left (respectively, right) ideal that have nonzero intersection. Therefore if $0 \neq a \in R$ then $R / R a$ is left Artinian and $R / a R$ is right Artinian.

\section{Ideal Theory and Divisibility in Noncommutative PIDs}

In this section we let $R$ denote a noncommutative PID. We present some of the basic features of ideal theory and divisibility in such a ring, using Jacobson's treatment, [26], as reference. We included proofs for some of the results, while in some cases we only indicated what is behind the proofs. When explanations are missing we invite the reader to consult the original work which is quite readable even today.

Two-sided ideals of $R$ will be simply referred to as ideals. A left (respectively, right) generator for an left (respectively, right) ideal $I$ is an element $a$ such that $I=R a$ (respectively, $I=a R$ ). Two elements $a, b$ in $R$ are called left associated if there exist $u, v \in R$ such that $a=u b, b=v a$. These conditions imply that $u v=v u=1$ if $a \neq 0$ or, equivalently, $b \neq 0$. Hence, for a left ideal in $R$, the left generators $a$ and $b$ for $I$ are left associated. Similarly, one can talk about right associated elements.

If $I$ is an ideal in $R$, then $I=R a=b R$ for some $a, b \in R$. Then $a=b u, b=v a$ for $u, v \in R$. This implies that $a=v a u, b=v b u$. Since $a u \in I$, then $a u=w a$, for some $w \in R$ and then we get $a=v a u=v w a$. So, $1=v w$ and this gives that $v$ is invertible. Also, similarly one gets that $u$ is invertible as well.

In conclusion $a$ is a left and right generator for $I$ (and so is $b$ ). Such an element $a$ with the property that $R a=a R$ is called a two-sided generator in $R$. So, any left (or right) generator of an ideal $I$ of $R$ is a two-sided generator.

Definition 3.1. Let $R$ be a PID and $a \in R$, nonzero and not a unit.

(i) We say that $a$ is irreducible if it cannot be written as the product of two nonzero, nonunits from $R$.

(ii) We say that $a$ is indecomposable if $R / R a$ is indecomposable as a left $R$-module. 
It is easy to see that $a$ is irreducible in $R$ if and only of $R / R a$ is nonzero simple left $R$-module.

Definition 3.2. Let $a, b \in R$ nonzero elements. The greatest left common divisor of $a, b$ is an element $d \in R$ such that $a=d a^{\prime}, b=d b^{\prime}$ and for any other element $d^{\prime} \in R$ with $a=d^{\prime} a^{\prime \prime}, b=d^{\prime} b^{\prime \prime}$ there exists $c \in R$ such that $d=d^{\prime} c$. We denote this element by $(a, b)_{l}$. In similar fashion one can define the least common right multiple of $a$ and $b$ which is denoted by $[a, b]_{r}$ : it is the element $m$ such that $m=x a=y b$ and for any other common right multiple $m^{\prime}$ of $a$ and $b$ there exists $z$ such that $m^{\prime}=z m$.

Furthermore, one can define a greatest right common divisor denoted by $(a, b)_{r}$ and the least left common multiple denoted by $[a, b]_{l}$.

Proposition 3.3. Let $a, b \in R$ nonzero. The greatest common left (respectively, right) divisor and the least common right (respectively, left) multiple exist and are nonzero and unique up to multiplication by units on the right (respectively, left).

An important notion for our purposes is that of similarity. It is defined as follows:

Definition 3.4. Let $a, b \in R$ both nonzero.

We say that $a$ and $b$ are left (respectively right) similar if and only if there exists $u \in R$ such that

$$
R=R u+R b, \quad R a u=R u \cap R b,
$$

(respectively, $R=u R+b R, u a R=u R \cap b R$.)

An important characterization of similarity that explains its relevance is given below.

Proposition 3.5. Let $a, b \in R$ both nonzero.

Then $a$ and $b$ are right similar if and only if they are left similar if and only if $R / R a \simeq R / R b$ as $R$-left modules if and only if $R / a R \simeq R / b R$ as $R$-right modules.

Proof. Assume that $a, b$ are left similar. Then the map $f: R / R a \rightarrow R / R b$ define by $f(c+R a)=c u+R b$ is well-defined and an isomorphism:

Indeed, $c-c^{\prime} \in R a$ implies $c u-c^{\prime} u \in R a u=R u \cap R b \subset R b$, so $c u+R b=$ $c^{\prime} u+R b$. Also, $c u \in R b$ implies that $c u \in R u \cap R b=R a u$ and so $c \in R a$. Finally, since $1=r u+s b$ for some $r, s \in R$ and therefore $c=c r u+c s b$ or $c=c r u \in R b$. Hence $f(c r+R a)=c r u+R b=c+R b$.

Now assume that that $f: R / R a \rightarrow R / R b$ is an isomorphism and let $u \in R$ such that $f(1+R a)=u+R b$. So $f(c+R a)=c(u+R b)=c u+R b$ for all $c \in R$. In particular, $a u \in R b$. So $R a u \subset R u \cap R b$. On the other hand, to show that $R u \cap R b \subset R a u$, note that if $c u \in R b$, then $f(c+R a)=0$ so $c \in R a$ because $f$ is injective. This shows that $c u \in R a u$ which is want we wanted. 
Finally, since $1+R b \in \operatorname{Im}(f)$ then there exists $c \in R$ such that $c u+R b=1+R b$. This immediately shows that $R=R u+R b$.

We also know that $a u=v b$ for some $v \in R$. We plan to show that $v r \cap a R=v b R$ and $v R+a R=R$ which shows that $a, b$ are right similar.

First let $z \in R$ such that $a R+v R=z R$. Then $a=z r, v=z s$ for some $r, s \in R$. Therefore, $z r u=z s b$ so $r u=s b \in R u \cap R b=R a u$ which shows that $r u=t a u$ or $r=t a=t z r$ and hence $1=t z$ Therefore $R=z R=v r+a R$.

Let $w \in R$ such that $v R \cap a R=w R$. Then since $a u=v b \in a R \cap v R=w R$ we see that $a u=v b=w s$ for some $s \in S$. But $w=v r=a t$, for some $r, t \in R$. In conclusion, $v b=v r s, a u=a t s$ and so $b=r s$ and $u=t s$. This gives that $R=R b+R u \subset R s$. Hence $s$ is invertible and then, because $v b=w s$, we get that $v b R=w R$. In conclusion, $v R \cap a R=v b R$.

Reversing the roles of left and right we see now that the result follows.

Therefore elements that are left (or right) similar will be called similar elements. An application of the Jordan-Hölder theorem gives the following result.

Proposition 3.6. Let $a \in R$ be a nonzero and not a unit. Then $a=b_{1} \cdots b_{n}$ with $b_{i}$ irreducible. If $a=c_{1} \cdots c_{m}$ with $c_{i}$ irreducible, then $m=n$ and the $b$ 's and $c$ 's may be be paired in similar pairs.

The number $n$ will be called the length of $a$ and denoted by $\lambda(a)$. It is also equal to the length of a composition series for $R / R a$. The following relation can be established.

Proposition 3.7. For any $a, b \in$ nonzero and nonunits we have that

$$
\lambda(a b)=\lambda\left([a, b]_{r}\right)+\lambda\left((a, b)_{l}\right) .
$$

Proposition 3.8. Let $a \in R$ be nonzero and not a unit. Then $a=\left[b_{1}, \ldots, b_{n}\right]_{r}$ where each $b_{i}$ is indecomposable and

$$
\left(b_{i},\left[b_{1}, \ldots, b_{i-1}, b_{i+1}, \ldots, b_{n}\right]_{r}\right)_{l}=1 .
$$

In addition if $a=\left[c_{1}, \cdots, c_{m}\right]_{r}$, where each $c_{i}$ is indecomposable, and

$$
\left(c_{i},\left[c_{1}, \ldots, c_{i-1}, c_{i+1}, \ldots, c_{m}\right]_{r}\right)_{l}=1,
$$

then $m=n$ and $b$ 's and c's can be paired in similar pairs.

Proof. This is an application of the Krull-Schmidt Theorem.

Definition 3.9. Let $a, b \in R$. We say that $a$ is a total divisor of $b$ if there is a two-sided ideal $I$ such that $R b \subseteq I \subseteq R a$. We will write $\left.a\right|_{t} b$. 
Proposition 3.10 (Teichmüller's condition). Let $a, b \in R$. Then $a$ is a total divisor of $b$ if and only if $R b R \subseteq R a \cap a R$.

Proof. First assume that $R b R \subseteq R a \cap a R$.

Since $R b \subseteq R b R \subseteq R a$ and $R b R$ is a two-sided ideal we conclude that $\left.a\right|_{t} b$.

Now assume that $\left.a\right|_{t} b$.

Let $I$ be an ideal generated by $x$ such that $R b \subseteq I=R x=x R \subseteq R a$.

Clearly, $R b \subseteq x R$ implies that $R b R \subseteq x R \subseteq R a$.

Let $a R+x R=d R$, so $a u+x v=d$ for some $u, v \in R$. Since $x=w a$ for some $w \in R$ we have that

$$
w d=w a u+w x v=w a u+x w^{\prime} v=w a u+w a w^{\prime} v=w\left(a u+a w^{\prime} v\right)
$$

with $w^{\prime} \in R$ since $R x=x R$.

So, $d=a u+a w^{\prime} v$ which implies that $d \in a R$.

But then $I=R x=x R \subseteq d R \subseteq a R$.

In conclusion $I \subseteq R a \cap a R$ which implies that $R b R \subseteq R a \cap a R$.

Remark 3.11. We would like to observe that total divisibility is invariant under similarity, more precisely, if $\left.a\right|_{t} b, a$ and $c$ are similar, and $b$ and $d$ are similar, then $\left.c\right|_{t} d$. This is Theorem 14 on page 40 in [26].

Proposition 3.12. Let $R$ be a PID.

(i) If $I \subset J$ are two-sided ideals, then $I=J \cdot J^{\prime}$ where $J^{\prime}$ is a two-sided ideal.

(ii) If $I$ is a proper two-sided ideal then $I=J_{1} \cdots J_{n}$ where each $J_{i}$ is a two-sided maximal ideal.

(iii) Any two maximal two-sided ideals $I, J$ commute, that is $I J=J I$.

(iv) The two-sided ideals form a commutative semigroup under multiplication, and, for any proper two-sided ideal I, the factorization of I into a product of maximal two-sided ideals is unique.

\subsection{Examples in $K\{F\}$}

For an element $f \in R=K\{F\}$ the degree of $f$ is in fact equal to $\operatorname{dim}_{K}\left(\frac{R}{R f}\right)$, so the degree of an element of $R$ is an invariant under similarity. Proposition 3.6 shows that the degree of the irreducible elements in the factorization of $f$ depend only on $f$.

To construct elements similar to $f$, let $u \in R$ be a unit. Then $R u=R$ and hence $R u \cap R f=R f$ and $R=R u+R f$. Therefore any generator of $R f$ that can be written in the form $g u$ will provide an element $g \in R$ similar to $f$. But a generator for $R f$ is of the form $v f$ with $v$ unit, and so $v f=g u$ or $g=v f u^{-1}$. In conclusion an element of the form $v f w$ with $v, w$ units in $R$ is similar to $f$.

For a concrete example let us take $f=F^{n}-\lambda$ with $\lambda \in K$. For $w, u \in K$ and nonzero we get that $g=v w^{p^{n}} F^{n}-v w \lambda$ is similar to $f$. Hence, $g=F^{n}-w^{-p^{n}+1} \lambda$ 
are monic polynomials that are similar to $f$. This example shows that similar elements do not generate necessarily the same left (or right) ideals.

Proposition 3.13. Let $K$ be a perfect field.

(i) Assume that $K=\mathbb{F}_{p^{k}}$. Let $f=\sum_{i=0}^{n} a_{i} F^{i}$ with $0 \neq a_{n} \in K$. Let $\operatorname{Supp}(f)=$ $\left\{i: a_{i} \neq 0\right\}$. Then $f$ is a two-sided generator if and only if $k$ divides $\operatorname{gcd}(j-i$ : $i \leq j$ with $i, j \in \operatorname{Supp}(f)$ ) (naturally, if $\operatorname{Supp}(f)$ has one element, we assume that the condition is empty).

(ii) Assume that $K$ is not finite. Then every ideal $I$ is generated by $F^{n}$ for some $n \geq 0$.

Proof. (i) If $I=R f=f R$, then by degree considerations we have that for all $a \in K$, there exists $b \in K$ such that $a f=f b$. In fact, since $F^{n}$ generates an ideal for any $n$, as can easily be checked, this condition is in fact necessary and sufficient for $f$ to satisfy $R f=f R$.

The equality $a f=f b$ implies that for all $i=0, \ldots, n$

$$
a a_{i}=a_{i} b^{p^{i}}
$$

which in turn is equivalent to

$$
a=b^{p^{i}}
$$

for all $i \in \operatorname{Supp}(f)$ which, in conjunction with $K$ perfect, says that $b^{p^{i}}=b^{p^{j}}$ for all $b \in K$ and $i \leq j$ with $i, j \in \operatorname{Supp}(f)$.

This is indeed equivalent to $c=c^{p^{j-i}}$, for all $c \in K$ and $i \leq j$ with $i, j \in$ $\operatorname{Supp}(f)$. Since $K=\mathbb{F}_{p^{k}}$ we get that $\mathbb{F}_{p^{k}} \subseteq \mathbb{F}_{p^{j-i}}$, that is, $k \mid j-i$, for all $i \leq j$ with $i, j \in \operatorname{Supp}(f)$.

(ii) When $K$ is not finite, the proof above shows that the only possibility is that

$$
|\operatorname{Supp}(f)|=1,
$$

which represents the claim.

Remark 3.14. Let $K=\mathbb{F}_{p^{k}}$. For degree $n$ polynomials $f=\sum_{i=0}^{n} a_{i} F^{i}$ with $a_{0} \neq 0$, the condition in Proposition 3.13 is equivalent to $k \mid i$ for all $i \in \operatorname{Supp}(f)$. Therefore, any two-sided generator $f \in K\{F\}$ is a product between a power of $F$ and a polynomial with nonzero constant coefficient and only nonzero degrees divisible by $k$ in its support.

Example 3.15. Let $K=\mathbb{F}_{p^{2}}$. Then $f=F^{6}+F^{4}-F^{2}-1$ is a two-sided generator for $R=K\{F\}$ according to the above Proposition.

Proposition 3.16. Let $a, b \in R=K\{F\}$ such that $\left.a\right|_{t} b$. Assume that $K$ is perfect, not finite. Then $a=u F^{k}$ where $k \geq 0$ and $u \in K$. 
Proof. Since $\left.a\right|_{t} b$, there exists an ideal $I$ of $R$ such that $I \subseteq R a$. But $K$ is not finite so by Proposition 3.13 we get that $I$ is generated by $F^{l}$ for some $l \geq 0$.

Therefore $F^{l}=r a$ with $r \in R$.

It is clear that $F$ is irreducible in $R=K\{F\}$ so by the unique factorization theorem one has that $a=u F^{k}$ where $k \geq 0$.

Example 3.17. Over a finite field, there exist many interesting examples of pairs $a, b \in$ $K\{F\}$ such that $\left.a\right|_{t} b$. For example let $K=\mathbb{F}_{p^{2}}$ and let $a=F^{3}+F^{2}+F+1$ and $b=F^{7}+F^{6}+F^{5}+F^{4}-F^{3}-F^{2}-F-1$. According to the Proposition 3.13 neither $a$ nor $b$ are two-sided generators in $R$.

However $b=(F+1)\left(F^{6}+F^{4}-F^{2}-1\right)$ and

$$
F^{6}+F^{4}-F^{2}-1=\left(F^{3}-F^{2}+F-1\right)\left(F^{3}+F^{2}+F+1\right) .
$$

As seen earlier, $F^{6}+F^{4}-F^{2}-1$ is a two-sided generator, so $\left.a\right|_{t} b$.

\section{Matrix Transformations over Noncommutative PIDs}

In this section we are considering matrices with entires in a noncommutative PID $R$. The collection of $n \times m$ matrices with entries in $R$ will be denoted by $M_{n, m}(R)$.

First we would like to note that a noncommutative PID $R$ can be embedded in a division ring $Q$ as follows: consider pairs of the form $(a, b) \in R \times R \backslash\{0\}$ and let $(a, b) \equiv(c, d)$ if $[b, d]_{r}=b b_{1}=d d_{1}$ implies $a b_{1}=c d_{1}$. This defines an equivalence relation and the equivalence class of a pair $(a, b)$ will be denoted by $a / b$.

The addition of two classes is defined by $a / b+c / d=\left(a b_{1}+c d_{1}\right) / m$ where $b_{1}, d_{1}$ are such that $[b, d]_{r}=b b_{1}=d d_{1}$. The multiplication is defined as follows: $(a / b) \cdot(c / d)=\left(a b_{2}\right) /\left(d c_{2}\right)$ if $c \neq 0,[b, c]_{r}=b b_{2}=c c_{2}$. If $c=0$, we simply put $(a / b) \cdot(0 / d)=0 / d$. It is well known that $Q$ defined in this fashion is a division ring and that $R$ can be naturally embedded in $Q$.

Since $R$ can be embedded in a division ring then for two $n \times n$ matrices $U, V$ with entries in $R$ such that $U V=1$ we see that $V U=1$ as well.

Let $A, B$ be two $n \times r$ matrices with entries in $R$. We say that $A, B$ are associates if $B=U A V$ where $U, V$ are invertible matrices in $M_{n, n}(R)$, respectively $M_{r, r}(R)$.

Let $E_{i j}$ denote the square matrix that has 1 on the main diagonal and the position $(i, j)$, and zero for the rest.

The following represents a list of elementary transformations for a given matrix $A \in M_{n, r}(R)$ :

(i) Adding to the $i$ th column the $j$ th column of the matrix $A$ multiplied on the right by $q$. Similarly, adding to the $i$ th row the $j$ th row of the matrix $A$ multiplied on the left by $q$. The operations are $A\left(1+E_{j i} q\right)$, respectively $\left(1+E_{i j} q\right) A$. 
(ii) Interchanging the $i$ th and the $j$ th rows (columns) of $A$. This corresponds to $A\left(1+E_{i j}+E_{j i}-E_{i i}-E_{j j}\right)$, respectively $\left(1+E_{i j}+E_{j i}-E_{i i}-E_{j j}\right) A$.

(iii) Multiplying the $i$ th column (row) on the right (left) by a unit $u$. This corresponds to $A\left(1+(u-1) E_{i i}\right)$, respectively $\left(1+(u-1) E_{i i}\right) A$.

In addition to these transformations, given a matrix $A$ with row

$$
\left(c_{1}, \ldots, c_{i-1}, a, c_{i+1}, \ldots, c_{j-1}, b, c_{j+1}, \ldots, c_{n}\right)
$$

there is a matrix associated to $A$ of the form $A V$ where $V$ is invertible with row $i$ equal to

$$
\left(c_{1}, \ldots, c_{i-1}, d, c_{i+1}, \ldots, c_{j-1}, 0, c_{j+1}, \ldots, c_{n}\right),
$$

where $d=(a, b)_{l}$. A similar statement holds for the columns of $A$ with the new matrix of the form $U A$ where $U$ is invertible. Such transformation will be called special transformations.

We sketch here the construction of the matrix $V$ that can be used in a special transformation. Since $(a, b)_{l}=d$ one can find $p, q, r, s \in R$ such that $p a+q b=d$ and $r a+s b=0$ and $R r+R s=R$. The it can be checked that the matrix

$$
\left(\begin{array}{ll}
p & q \\
r & s
\end{array}\right)
$$

is invertible. Finally the matrix $U=\left(u_{i j}\right)_{1 \leq i, j \leq n}$ has the following entries: $u_{h h}=1$ for $h \neq i, j, u_{i i}=p, u_{j j}=s, u_{i j}=r, u_{j i}=q$ and the rest of the entries are zero.

Repeated application of elementary and special transformations leads to the following:

Theorem 4.1. Any matrix $A \in M_{n, r}(R)$ has an associate with nonzero elements $d_{1}, d_{2}, \ldots, d_{s}$ on the main diagonal only such that $s \leq \min (n, r)$ and $d_{i}$ is a total divisor of $d_{j}$ for $i<j$ :

$$
\left(\begin{array}{ccc}
d_{1} & 0 & \cdots \\
0 & d_{2} & \ddots \\
\vdots & \ddots & \ddots
\end{array}\right) .
$$

Proof. We will sketch here the procedure that gives an associate of $A$ as in the conclusion of the theorem. The idea is to use elementary transformations and therefore arrange that $A$ is associated to a matrix that has in the upper left corner a nonzero element of smallest length. If this element $a_{11}$ is not a left factor of any of the $a_{1 i}$ (or a right factor of any of the $a_{j 1}$ ) then after elementary transformations one can replace $a_{11}$ by an element of smaller length. After a finite number of elementary transformations, we therefore obtain an element in the upper left corner that is a left factor (respectively, right factor) of any element in the first row (respectively, first column). 
One can perform repeated elementary transformations to obtain an associated matrix to $A$ that has a nonzero entry in the upper left corner and zeros on the rest of the entries on the first row and first column. Continuing like this we produce an associated matrix to $A$ that has only nonzero entries on the diagonal, say $d_{1}, d_{2}, \ldots, d_{s}$.

Let us show that we may assume that $\left.d_{i}\right|_{t} d_{j}$ for all $i<j$. In the case that there exists $b \neq 0$ such that $d_{i}$ is not a left factor of $d_{j}$, go ahead and rename $i=1, j=2$ for simplicity. The upper left corner of the matrix is of the form

$$
\left(\begin{array}{cc}
d_{1} & b d_{2} \\
0 & d_{2}
\end{array}\right)
$$

after an elementary transformation. The highest left factor of $d_{1}$ and $b d_{2}$ has length less that the length of $d_{1}$. We can repeat the diagonalization process to obtain an associated matrix with an upper left corner entry of length smaller than the length of $d_{1}$. Repeated application of this process produces an associated diagonal matrix where $d_{i}$ is a left factor for all $b d_{j}$ for all nonzero $b$ and all $i<j$. But then obviously $R d_{j} \subset R d_{j} R \subset d_{i} R$ which shows that $\left.d_{i}\right|_{t} d_{j}$.

\section{Module Theory over Noncommutative PIDs}

Any cyclic $R$-module $M$ is isomorphic to $R / R a$ where $a \in R$. If $M=R m$, then $R a=\operatorname{Ann}_{R}(m)=\{r \in R: r m=0\}$. We call the element $m$ torsion if $\operatorname{Ann}_{R}(m) \neq 0$. Since in this case $\operatorname{Ann}_{R}(m)=R \mu$ for some $0 \neq \mu \in R$ we say that $\mu$ is the $\operatorname{order}$ of $m$ and denoted by $\operatorname{ord}(m)$. Any two orders of $m$ are left associated.

Let $M$ be an $R$-module. It can be checked that $T(M)=\{m \in M: \operatorname{ord}(m) \neq 0\}$ is a submodule of $M$. The reader that has difficulty verifying this statement will find helpful the observation that the intersection of two left nonzero ideals is a nonzero left ideal. As always, if $M=T(M)$ we say that $M$ is a torsion $R$-module.

The following result follows from the Krull-Schmidt Theorem for modules (p. 115 in [27]).

Proposition 5.1. Let $M$ be an R-module that satisfies the (ACC) and (DCC) conditions on its left submodules. Then

$$
M \simeq I_{1} \oplus \cdots \oplus I_{k}
$$

where $I_{l}$ are indecomposable $R$-modules for all $l=1, \ldots, k$. For any other similar decomposition into indecomposable $R$-modules

$$
M \simeq L_{1} \oplus \cdots \oplus L_{h}
$$

we have $k=h$ and, up to renumbering, $I_{l} \simeq L_{l}$ for all $l=1, \ldots, k$. 
In what follows we will need some basic considerations on linear maps of free modules over a ring $R$ and matrices with entries in $R$. We will of course use this in the noncommutative setting, so we will review these facts in this case as many basic books discuss them only in the special commutative case.

Let $\phi: R^{m} \rightarrow R^{n}$ be an $R$-linear map. Fix $u_{1}, \ldots, u_{m}$ and $v_{1}, \ldots, v_{n}$ be bases of $R^{m}$, respectively $R^{n}$, and write $\phi\left(u_{i}\right)=\sum_{j=1}^{n} a_{i j} v_{j}$ for all $i=1, \ldots, m$. Then if $r=\sum_{i=1}^{m} r_{i} u_{i}$, then $\phi(r)=\sum_{i=1}^{m} r_{i} \phi\left(u_{i}\right)=\sum_{j=1}^{n} \sum_{i=1}^{m}\left(r_{i} a_{i j}\right) v_{j}$. If we set $A=\left(a_{i j}\right)_{1 \leq i \leq n, 1 \leq j \leq m}$, the map $\phi$ can be described by

$$
\left(r_{1}, \ldots, r_{n}\right) \longmapsto\left(r_{1}, \ldots, r_{n}\right) A .
$$

A simple check establishes the existence of a group isomorphism

$$
\Theta:\left(\operatorname{Hom}_{R}\left(R^{m}, R^{n}\right),+\right) \longrightarrow\left(M_{m, n}(R),+\right)
$$

given by

$$
\phi \longmapsto A \text {. }
$$

Obviously, the map $\Theta=\Theta_{u, v}(\phi)$ depends on the bases $u=\left\{u_{1}, \ldots, u_{m}\right\}$ and $v=\left\{v_{1}, \ldots, v_{n}\right\}$, and we will not indicate the chosen bases, when the choice is clear from the context. The construction of a well-defined $\Theta_{u, v}(\phi)$ requires that $v$ is a basis for $R^{n}$, while $u$ only needs to be a set of generators for $R^{m}$.

Now consider another $R$-linear map $\psi: R^{n} \rightarrow R^{h}$ and let $w_{1}, \ldots, w_{h}$ be a basis for $R^{h}$. Let $\psi\left(v_{j}\right)=\sum_{l=1}^{h} b_{j l} w_{l}$, for all $j=1, \ldots, n$. Therefore

$$
\begin{aligned}
(\psi \circ \phi)(r) & =\sum_{i=1}^{m} r_{i}(\psi \circ \phi)\left(u_{i}\right) \\
& =\sum_{i=1}^{m} r_{i} \psi\left(\sum_{j=1}^{n} a_{i j} v_{j}\right) \\
& =\sum_{i=1}^{m} r_{i} \sum_{j=1}^{n} a_{i j} \psi\left(v_{j}\right) \\
& =\sum_{i=1}^{m} r_{i}\left(\sum_{j=1}^{n} a_{i j} b_{j l}\right) w_{l} .
\end{aligned}
$$

This computation shows that

$$
\Theta_{u, w}(\psi \circ \phi)=A B=\Theta_{u, v}(\phi) \cdot \Theta_{v, w}(\psi) .
$$

Now consider two bases for $R^{n}, e=\left\{e_{1}, \ldots, e_{n}\right\}$ and $f=\left\{f_{1}, \ldots, f_{n}\right\}$. Write $e_{i}=\sum_{j=1}^{n} t_{i j} f_{j}$ and denote $T=\left(t_{i j}\right)_{1 \leq i, j \leq n}$, an $n \times n$-matrix with entries from $R$, referred to as the transition matrix between bases. 
This can be written in matrix form as

$$
e=\left(\begin{array}{c}
e_{1} \\
e_{2} \\
\vdots \\
e_{n}
\end{array}\right)=T \cdot\left(\begin{array}{c}
f_{1} \\
f_{2} \\
\vdots \\
f_{n}
\end{array}\right)=T \cdot f .
$$

It is clear that $\Theta_{e, f}\left(1_{R^{n}}\right)=T$. Similarly we can write $f=S \cdot e$ where $S$ is an $n \times n$ matrix with entries in $R$. In particular, $I_{n}=\Theta_{e, e}\left(1_{R^{n}}\right)=\Theta_{e, f}\left(1_{R^{n}}\right) \cdot \Theta_{f, e}\left(1_{R^{n}}\right)=$ $T S$. Similarly, $S T=I_{n}$.

Now let $\phi: R^{m} \rightarrow R^{n}$ and we fix bases $u=\left\{u_{1}, \ldots, u_{m}\right\}$ and $v=\left\{v_{1}, \ldots, v_{n}\right\}$. Assume that $u^{\prime}=\left\{u_{1}^{\prime}, \ldots, u_{m}^{\prime}\right\}$ and $v^{\prime}=\left\{v_{1}^{\prime}, \ldots, v_{n}^{\prime}\right\}$ are bases for $R^{m}$ and $R^{n}$ and let $C, D$ be the transition matrices such that $u^{\prime}=C u, v^{\prime}=D v$. Note that $\Theta_{u^{\prime}, u}\left(1_{R^{m}}\right)=C$ and $\Theta_{v, v^{\prime}}\left(1_{R^{n}}\right)=D^{-1}$.

Then $\Theta_{u^{\prime}, v}(\phi)=\Theta_{u^{\prime}, v}\left(1_{R^{m}} \phi\right)=\Theta_{u^{\prime}, u}\left(1_{R^{m}}\right) \Theta_{u, v}(\phi)=C \Theta_{u, v}(\phi)$, and, similarly, $\Theta_{u, v}(\phi)=\Theta_{u, v^{\prime}}(\phi) D$.

Using this, we can see that $\Theta_{u^{\prime}, v^{\prime}}(\phi)=C \Theta_{u, v}(\phi) D^{-1}$.

The following two results have proofs similar to their well-known counterparts in the commutative case and are not included below (see Theorems 17 and 18 in [26]).

Theorem 5.2. Let $G$ be a free $R$-module that admits a basis with $n$ elements and $N \subset G$ a submodule. Then $N$ is also free and there exists a basis of $N$ with $m \leq n$ elements.

Theorem 5.3. Let $M$ be a finitely generated $R$-module. Then there exists a finite rank free submodule $G$ of $M$ such that

$$
M=T(M) \oplus G
$$

Theorem 5.4. If $M$ is a finitely generated torsion $R$-module, then there exist nonzero elements $f_{1}, \ldots, f_{n}$ in $R$ such that $f_{i}$ is a total divisor of $f_{i+1}$ for all $i=1, \ldots, n-1$ and

$$
M \simeq \frac{R}{R f_{1}} \oplus \cdots \oplus \frac{R}{R f_{n}} .
$$

Proof. Since $M$ is finitely generated over $R$ we can map a free $R$-module onto $M$ via an $R$-module homomorphism $\phi, \phi: R^{n} \rightarrow M \rightarrow 0$. Let $N=\operatorname{Ker}(\phi)$. According to Theorem 5.2, $N$ admits a basis $f_{1}, \ldots, f_{m}$ with $m$ elements, where $m \leq n$. Denote $e=\left\{e_{1}, \ldots, e_{n}\right\}$ the standard basis in $R^{n}$.

Therefore $M=\operatorname{Coker}\left(i: N \hookrightarrow R^{n}\right)$. Let $\Theta(i)=A$ and note that according to our earlier observations, a change of bases for $N$ and $R^{n}$ to say $f^{\prime}$, respectively $e^{\prime}$ produces a new matrix $\Theta_{f^{\prime}, e^{\prime}}(i)$ that is associate of $A$. 
Therefore we can apply the matrix considerations from an earlier section and conclude that for an appropriate choice of bases for $N$ and $R^{n}$ we have

$$
\Theta(i)=\left(\begin{array}{ccc}
f_{1} & 0 & \cdots \\
0 & f_{2} & \ddots \\
\vdots & \ddots & \ddots
\end{array}\right)
$$

with $f_{i}$ is a total divisor for $f_{j}$ for all $j>i$. Let $s$ the number of nonzero $f_{i}$ 's. Clearly $s \leq n$. Since $\operatorname{Coker}(\Theta(i))=R / R f_{1} \oplus R / R f_{2} \oplus \cdots \oplus R / R f_{s} \oplus R^{n-s}$, we can conclude that $s=n$ since otherwise $M$ has nontorsion elements. The statement of the theorem is now clear.

Corollary 5.5. (i) Let $M$ be a finitely generated indecomposable R-module. Then $M$ is cyclic.

(ii) Any R-module $M$ that satisfies the (ACC) and (DCC) conditions on its left submodules is a direct sum of cyclic indecomposable $R$-modules of the form $R / R m$, $m \in R$. The elements $m$ that appear in the decomposition are determined up to similarity and their number is an invariant of the module $M$.

(iii) If $M$ is finitely generated $R$-torsion module, then there exist $n$ and nonzero $e_{1}, \ldots, e_{n}$ such that for all $i=1, \ldots, n, R / R e_{i}$ is indecomposable and

$$
M \simeq R / R e_{1} \oplus \cdots \oplus R / R e_{n} .
$$

The number $n$ is uniquely determined by $M$ and the elements $e_{1}, \ldots, e_{n} \in R$ are determined up to similarity.

The elements in the above Corollary are called elementary divisors of $M$, in the case $M$ is $R$-torsion and finitely generated. In the general case when $M$ is finitely generated, but not necessarily $R$-torsion, the elementary divisors of $M$ are by definition the elementary divisors of $T(M)$.

\section{Computing the Invariant Factors}

Let $F: V \rightarrow V$ be a Frobenius action on $V$. Let $\left\{v_{1}, \ldots, v_{n}\right\}$ denote a basis for $V$ and write

$$
F\left(v_{i}\right)=\sum_{j=1}^{n} a_{i j} v_{j}, \quad i=1, \ldots, n, a_{i j} \in K .
$$

Therefore

$$
F\left(\sum_{i=1}^{n} x_{i} v_{i}\right)=\sum_{i=1}^{n} x_{i}^{p} F\left(v_{i}\right)=\sum_{i=1}^{n} x_{i}^{p} \sum_{j=1}^{n} a_{i j} v_{j}=\sum_{j=1}^{n}\left(\sum_{i=1}^{n} x_{i}^{p} a_{i j}\right) v_{j} .
$$


If we write $\left(x_{1}, \ldots, x_{n}\right)$ for $\sum_{j=1}^{n} x_{j} v_{j}$ and $A=\left(a_{i j}\right)_{1 \leq i, j \leq n}$ then the above equation can be summarized as

$$
F\left(x_{1}, \ldots, x_{n}\right)=\left(x_{1}^{p}, \ldots, x_{n}^{p}\right) \cdot A
$$

The $R=K\{F\}$-structure on $V$ is defined by $F \cdot v=F(v)$. So given a fixed basis $\left\{v_{1}, \ldots, v_{n}\right\}$ of $V$, the action $F$ is determined by the matrix $A$ and vice versa. We would like to outline the computation of the invariant factors of $V$ as an $R$-module from the matrix $A$.

Note that we can define a $R$-module homomorphism $\phi: R^{n} \rightarrow V$ by letting $\phi\left(e_{i}\right)=v_{i}$ where $e=\left\{e_{1}, \ldots, e_{n}\right\}$ form a basis for $R^{n}$. This is obviously a surjection since $V$ is generated over $K$ and hence over $K\{F\}$ by $v_{1}, \ldots, v_{n}$.

Let us notice that $V$ is torsion over $R$. Indeed, since $\operatorname{dim}_{K}(V)<\infty$, for any $v \in V$, there exists $n$ such that $v, F(v), \ldots, F^{n}(v)$ are $K$-linearly dependent and so there exist $a_{0}, \ldots, a_{n}$ in $K$, not all zero, such that $a_{0} v+a_{1} F(v)+\cdots+a_{n} F^{n}(v)=0$. One can assume that $a_{n} \neq 0$ and note that this implies that $\left(a_{0}+a_{1} F+\cdots+a_{n} F^{n}\right) \cdot v=0$. In conclusion, Theorem 5.4 can be applied.

Theorem 6.1. Let $V$ be a $K$-vector space with basis $\left\{v_{1}, \ldots, v_{n}\right\}$. Assume that $V$ admits a Frobenius action $F$ over $K$ and let $A$ be the matrix associated to this action with respect to the chosen basis.

Consider the natural R-homomorphism $\phi: R^{n} \rightarrow V$ sending each element $e_{i}$ of the basis to $v_{i}$ for all $i=1, \ldots, n$. Then a basis for $W=\operatorname{Ker}(\phi)$ is given by the set of vectors

$$
\left(F I_{n}-A\right) \cdot\left(\begin{array}{c}
e_{1} \\
e_{2} \\
\vdots \\
e_{n}
\end{array}\right)
$$

(interpreted as a matrix multiplication).

Proof. Let

$$
\left(\begin{array}{c}
f_{1} \\
f_{2} \\
\vdots \\
f_{n}
\end{array}\right)=\left(F \cdot 1_{n}-A\right) \cdot\left(\begin{array}{c}
e_{1} \\
e_{2} \\
\vdots \\
e_{n}
\end{array}\right)
$$

therefore

$$
f_{i}=F \cdot e_{i}-\sum_{j=1}^{n} a_{i j} e_{j}
$$

Note that $\phi\left(f_{i}\right)=F \phi\left(e_{i}\right)-\sum_{j=1}^{n} a_{i j} \phi\left(e_{j}\right)=F\left(v_{i}\right)-\sum_{j=1}^{n} a_{i j} v_{j}=0$ which shows that each $f_{i}$ belongs to $W$. 
To prove that $f_{1}, \ldots, f_{n}$ generate $W$ let us denote by $W^{\prime}$ the $R$-submodule generated by these elements. The goal is to check that $W^{\prime}=W$. Consider the $K$-subspace of $R^{n}, E$, generated by $e_{1}, \ldots, e_{n}$. Clearly, $F \cdot e_{i}=f_{i}+\sum_{j=1}^{n} a_{i j} e_{j}$ and so belongs to $W^{\prime}+E$. Using this fact, we can immediately check that $W^{\prime}+E$ is an $R$-submodule of $R^{n}$. Now, it contains all $e_{1}, \ldots, e_{n}$ so it must equal $R^{n}$. Let $w \in W \subset R^{n}$ and write $w=w^{\prime}+e$ with $w^{\prime}=\sum_{i=1}^{n} r_{i} f_{i}$ and $e=\sum_{i=1}^{n} a_{i} e_{i}$ where each $r_{i} \in R$ and $a_{i} \in K$. But $\phi(w)=0$. We already know that $\phi\left(w^{\prime}\right)=0$, so we obtain $\phi(e)=0$ which leads to $\sum_{i=1}^{n} a_{i} \phi\left(e_{i}\right)=\sum_{i=1}^{n} a_{i} v_{i}=0$ therefore $a_{i}=0$ for all $i=1, \ldots, n$. Hence $w=w^{\prime} \in W^{\prime}$ and the equality $W^{\prime}=W$ is verified.

To check that $f_{1}, \ldots, f_{n}$ are linearly independent over $R$ let us consider $r_{1}, \ldots, r_{n} \in$ $R$ such that

$$
r_{1} f_{1}+r_{2} f_{2}+\cdots+r_{n} f_{n}=0 .
$$

Now each nonzero $r_{i}$ has a leading term of the form $a_{n_{i}} F^{n_{i}}$ with $a_{n_{i}} \in K, a_{n_{i}} \neq 0$. Consider the above relation in the highest degree for which $F$ appears on the left hand side, and note that it leads to a relation of the form $\sum_{i} a_{n_{i}} e_{i}=0$, where the sum runs over a nonempty subset of the set of indices $i$ such that $r_{i} \neq 0$. But $e_{1}, \ldots, e_{n}$ are linearly independent over $K$, so in fact we must have $r_{i}=0$ for all $i$.

Corollary 6.2. Let $V$ be a $K$-vector space with basis $\left\{v_{1}, \ldots, v_{n}\right\}$. Assume that $V$ admits a Frobenius action $F$ over $K$ and let $A$ be the matrix associated to this action with respect to the chosen basis.

Then the invariant factors of $V$ as an $R$-module are the invariant factors of $F \cdot 1_{n}-A$.

Proof. Construct a surjection $\phi: R^{n} \rightarrow V$ by mapping the elements of a basis $e=$ $\left\{e_{1}, \ldots, e_{n}\right\}$ to $V$ by $\phi\left(e_{i}\right)=v_{i}$. Let $W=\operatorname{Ker}(\phi)$.

In the proof of Theorem 5.4 the invariant factors of $V$ are obtained as the invariant factors of the matrix $\Theta(i)$ where $i: W \rightarrow V$ is the natural inclusion.

With the notations from Theorem 6.1, we have that $f=\left\{f_{1}, \ldots, f_{n}\right\}$ is basis of $W$. But $f_{i}=F \cdot e_{i}-\sum_{j=1}^{n} a_{i j} e_{j}$ and so $\Theta_{f, e}(i)=F \cdot 1_{n}-A$.

Let us present a example.

Example 6.3. Let $K$ be a field of characteristic $p>2, V=K^{2}$ and denote $v_{1}=$ $(1,0), v_{2}=(0,1)$ for the canonical basis for $V$.

Let $A=\left(\begin{array}{cc}1 & 0 \\ -2 & 0\end{array}\right)$ and define a Frobenius action on $V$ such that its matrix with respect to the chosen basis equals $A$, that is $F\left(v_{1}\right)=v_{1}, F\left(v_{2}\right)=-2 v_{1}$.

For arbitrary $(x, y) \in V$, we have $F(x, y)=\left(x^{p}, y^{p}\right) \cdot\left(\begin{array}{cc}1 & 0 \\ -2 & 0\end{array}\right)=\left(x^{p}-2 y^{p}, 0\right)$.

Consider now

$$
F \cdot I_{n}-A=\left(\begin{array}{cc}
F-1 & 0 \\
2 & F
\end{array}\right) \text {. }
$$

We will perform the elementary and special transformations described in Section 4 in order to compute the invariant factors. An important feature is that we can multiply 
a rown on the left and add to another row, and we can multiply a column on the right and add it to another column as described in Section 5. The rows will be denoted by $R_{1}, R_{2}$ and columns by $C_{1}, C_{2}$ and the transformations will be described as follows: $R_{1}^{\prime}=R_{1}+a R_{2}$ means for example that the we replace the old row $R_{1}$ by a new one obtained by first multiplying row $R_{2}$ on the left by $a$ and then adding it to row $R_{1}$. Therefore

$$
\left(\begin{array}{cc}
F-1 & 0 \\
2 & F
\end{array}\right) \stackrel{R_{1}^{\prime}=\left(-1 / 2^{p}\right) F R_{2}+R_{1}}{\sim}\left(\begin{array}{cc}
-1 & \left(-1 / 2^{p}\right) F^{2} \\
2 & F
\end{array}\right)
$$

and

$$
\left(\begin{array}{cc}
-1 & \left(-1 / 2^{p}\right) F^{2} \\
2 & F
\end{array}\right) \stackrel{R_{2}^{\prime}=2 R_{1}+R_{2}}{\sim}\left(\begin{array}{cc}
-1 & \left(-1 / 2^{p}\right) F^{2} \\
0 & F-\left(2 / 2^{p}\right) F^{2}
\end{array}\right)
$$

Continuing like this we obtain

$$
\left(\begin{array}{cc}
-1 & \left(-1 / 2^{p}\right) F^{2} \\
0 & F-\left(2 / 2^{p}\right) F^{2}
\end{array}\right) C_{2}^{\prime}=C_{1}\left(-1 / 2^{p}\right) F^{2}+C_{2}\left(\begin{array}{cc}
-1 & 0 \\
0 & F-\left(2 / 2^{p}\right) F^{2}
\end{array}\right)
$$

and further

$$
\left(\begin{array}{cc}
-1 & 0 \\
0 & F-\left(2 / 2^{p}\right) F^{2}
\end{array}\right) \sim\left(\begin{array}{cc}
\left(1 / 2^{p-1}\right) F^{2}-F & 0 \\
0 & 1
\end{array}\right)
$$

Therefore $V$ is $R$-isomorphic to

$$
\frac{R}{\left(\left(1 / 2^{p-1}\right) F^{2}-F\right) R},
$$

and we can understand better the action of $F$ on $V$ by inspecting the elementary transformations performed on $F \cdot I_{n}-A$.

These elementary transformations correspond to multiplying $F \cdot I_{n}-A$ on the left by

$$
X=\left(\begin{array}{cc}
0 & 1 \\
-1 & 0
\end{array}\right) \cdot\left(\begin{array}{ll}
1 & 0 \\
2 & 1
\end{array}\right) \cdot\left(\begin{array}{cc}
1 & \left(-1 / 2^{p}\right) F \\
0 & 1
\end{array}\right)
$$

and on the right by

$$
Y=\left(\begin{array}{cc}
1 & \left(-1 / 2^{p}\right) F^{2} \\
0 & 1
\end{array}\right) \cdot\left(\begin{array}{cc}
0 & 1 \\
-1 & 0
\end{array}\right)
$$

But

$$
X=\left(\begin{array}{cc}
2 & \left(-1 / 2^{p-1}\right) F+1 \\
-1 & \left(1 / 2^{p}\right) F
\end{array}\right)
$$

while

$$
Y=\left(\begin{array}{cc}
\left(1 / 2^{p}\right) F^{2} & 1 \\
-1 & 0
\end{array}\right)
$$


We know that $V=\operatorname{Coker}(i)$ where $i: W \rightarrow R^{2}$, and as in the proof of Theorem 5.4 the matrix

$$
\left(\begin{array}{cc}
\left(1 / 2^{p-1}\right) F^{2}-F & 0 \\
0 & 1
\end{array}\right)
$$

is the matrix of the map $i$ with respect to the basis $X \cdot f$ for $W$ and the basis $Y^{-1} \cdot e$ for $R^{2}$.

Now the

$$
Y^{-1} \cdot e=\left(\begin{array}{cc}
0 & -1 \\
1 & \left(1 / 2^{p}\right) F^{2}
\end{array}\right) \cdot\left(\begin{array}{l}
e_{1} \\
e_{2}
\end{array}\right)=\left(\begin{array}{c}
-e_{2} \\
e_{1}+\left(1 / 2^{p}\right) F^{2} e_{2}
\end{array}\right) .
$$

Under the isomorphism

$$
\frac{R}{\left(\left(1 / 2^{p-1}\right) F^{2}-F\right) R} \simeq V
$$

the class of the element 1 on the left corresponds to the image of the class of $-e_{2}$ in $V=\operatorname{Coker}(i)$ on the right therefore. So 1 corrresponds to $-v_{2}$.

Note that $\frac{R}{\left(\left(1 / 2^{p-1}\right) F^{2}-F\right) R}$ has $K$-basis $w_{1}=1, w_{2}=F$. The reader might want to know what basis of $V$ this is. Our above calculations have shown that 1 corresponds to $-v_{2}$ and, of course, $F$ will correspond to $F\left(-v_{2}\right)=(-1)^{p} F\left(v_{2}\right)=$ $-\left(-2 v_{1}\right)=2 v_{1}$.

In particular we obtain that $V$ is $R$-generated by $-v_{2}$ and $\operatorname{Ann}_{R}\left(-v_{2}\right)=\left(F^{2}-\right.$ $\left.2^{p-1} F\right)$.

\subsection{Injective Frobenius Actions on Finite Dimensional Vector Spaces over a Perfect Field}

In the study of modules with Frobenius action the case when the action is injective plays an especially important role.

Proposition 6.4. Let $K$ be a perfect field and $V$ be a finitely dimensional vector space with a Frobenius action $F$. Fix a basis for $V$ say $v=\left\{v_{1}, \ldots, v_{n}\right\}$ and denote by $A$ the matrix associated to $F$. Then $F$ is injective if and only if $A$ is invertible in $M_{n}(K)$.

Proof. The proof is a simple exercise.

The following theorem, that we could not find in the literature, is a simple consequence to the theory developed so far.

Theorem 6.5. Let $K$ be an infinite perfect field and $V$ be a finitely dimensional $K$ vector space. Assume that $V$ admits an injective Frobenius action. Then $V$ is $R$-cyclic. 
Proof. We have noted earlier that $V$ is necessarily a torsion $R$-module. Therefore $V$ is $R$-isomorphic to a direct sum of the form $\bigoplus_{i=1}^{S} R / R f_{i}$, where $f_{1}, \ldots, f_{s}$ are the invariant factors of $V$ regarded as an $R$-module.

The invariant factors $f_{1}, \ldots, f_{s}$ have the property $\left.f_{i}\right|_{t} f_{i+1}, i=1, \ldots, s-1$. This implies that there exist ideals $I_{i}$ such that $R f_{i} \subset I \subset R f_{i+1}$. However, ideals in $R$ are of the form $\left(F^{k}\right)$ for some $k$ by Proposition 3.13. So let $I_{i}=\left(F^{k_{i}}\right)$ for all $i=1, \ldots, s-1$. Since $F^{k_{i}} \in R f_{i+1}$, and $f_{i+1}$ kills a nonzero element of $V$ as can be readily seen from the $R$-structure of $V$, we see that $F^{k_{i}}$ kills a nonzero element of $V$. But $F$ is an injective action on $V$ so we conclude that $f_{i}=0$ for all $i \geq 2$ which leads to the conclusion of the theorem.

We can use this theorem to give a proof of a result of Dieudonné. This result is well-known and has appeared in many texts.

Theorem 6.6 (Dieudonné). Let $K$ be an algebraically closed field and $V$ a finite dimensional $K$-vector space with an injective Frobenius action $F_{K}: V \rightarrow V$. Then $V$ admits a K-basis which is fixed under $F$.

We will give an extension here that is suited to our point of view and it has been known to the experts. For example, the reader might consult [4] (Section 3.6.1) for a nice proof that has some similarities with the one below.

Theorem 6.7. Let $K$ be a perfect field and $V$ a finite dimensional $K$-vector space with an injective Frobenius action $F_{K}: V \rightarrow V$. Then there exists a finite field extension $K \subset L$ such that the Frobenius action on $V_{L}=L \otimes_{K} V$ given by

$$
F_{L}: V_{L} \rightarrow V_{L}, \quad F_{L}=1_{L} \otimes F_{K}
$$

admits an L-basis which is fixed under $F_{L}$.

Proof. According to Theorem 6.5 we know that $V=R / R f$ where $f \in R=K\{F\}$. We can assume that $f=F^{n}-\sum_{i=1}^{n} \lambda_{i} F^{i-1}$, with $\lambda_{1} \neq 0$ because $F$ acts injectively on $V$.

Let us fix the following basis of $V: v_{i}=F^{i-1}, i=1, \ldots, n$. With respect to this basis the matrix of the Frobenius action is given by

$$
A=\left(\begin{array}{ccccc}
0 & 1 & 0 & \cdots & 0 \\
0 & 0 & 1 & \ddots & \vdots \\
\vdots & \vdots & \ddots & \ddots & 0 \\
0 & 0 & \cdots & 0 & 1 \\
\lambda_{1} & \lambda_{2} & \lambda_{3} & \cdots & \lambda_{n}
\end{array}\right) .
$$

If $v=x_{1} v_{1}+\cdots+x_{n} v_{n}$, then $F(v)=v$ leads to the following system of equations

$$
x_{i}^{p}+x_{n}^{p} \lambda_{i+1}=x_{i+1}, \quad i=0, \ldots, n-1, \quad x_{0}=0 .
$$


Substituting recursively we obtain $\sum_{i=1}^{n} x_{n}^{p^{n-i+1}} \lambda_{i}^{p^{n-i}}-x_{n}=0$ which is a polynomial over $K$ admitting distinct roots, because its derivative is invertible. Call these roots $x_{1, n}, x_{2, n}, \ldots, x_{p^{n}, n}$. Adjoining these solutions to $K$ we get a finite field extension $L$ of $K$.

Once $x_{n}=x_{k, n}$ is obtained we see that the corresponding $x_{1}, \ldots, x_{n-1}$ can be calculated immediately with $x_{j}=x_{k, j}=\sum_{i=1}^{j} x_{k, n}^{p^{n-i+1}} \lambda_{i}^{p^{n-i}}$.

We claim that for $n$ distinct nonzero choices of $x_{k, n}, k=1, \ldots, n$, the elements of $V_{L}$ given by

$$
w_{j}=x_{j, 1} v_{1}+\cdots+x_{j, n} v_{n}
$$

with $j=1, \ldots, n$ form a basis for $V_{L}$. It is clear that $F_{L}\left(w_{j}\right)=w_{j}$.

The transition matrix from basis $v_{1}, \ldots, v_{n}$ to basis $w_{1}, \ldots, w_{n}$ has $k$ th row equal to

$$
\left(x_{k, n}^{p} \lambda_{1}, \ldots, \sum_{i=1}^{j} x_{k, n}^{p^{n-i+1}} \lambda_{i}^{p^{n-i}}, \ldots\right) .
$$

We need to show that this matrix has nonzero determinant. Since $\lambda_{1} \neq 0$, we can in fact assume $\lambda_{1}=1$, and now simple column operations show that the determinant of the transition matrix is a Vandermonde determinant in the nonzero distinct elements $x_{1, n}, \ldots, x_{n, n}$, which is therefore different from zero.

Definition 6.8. Let $V$ be a $K$-vector space with a Frobenius action. For a subset $S$ of $V,\langle S\rangle_{K}$ denotes the $K$-subspace of $V$ generated by the elements of $S$. Then the stable part of $V$ is the subspace $V_{s}=\bigcap_{e \geq 0}\left\langle F^{e}(V)\right\rangle_{K}$. The nilpotent part of $V$ is the subspace $V_{\text {nil }}=\left\{v \in V: F^{e}(v)=0\right.$ for some $\left.e \geq 0\right\}$. It is clear that both $V_{s}$ and $V_{\text {nil }}$ inherit the Frobenius action from $V$. Also, $V$ is called semisimple if $\langle F(V)\rangle_{K}=V$. Therefore $V_{s}$ is semisimple.

Proposition 6.9. Let $K$ be perfect field. Let $V$ be a $K$-vector space with a Frobenius action. Then $V=V_{s} \oplus V_{\text {nil }}$.

Proof. Since $K$ is perfect then $\left\langle F^{e}(V)\right\rangle_{K}=F^{e}(V)$ for all $e \geq 0$. There exists $e_{0} \geq 0$ such that $V_{s}=F^{e}(V)$ for all $e \geq e_{0}$. Also since $V_{\text {nil }}$ is finite dimensional we can find $m \geq 0$ such that $F^{m}\left(V_{\text {nil }}\right)=0$. One can assume $m \geq e_{0}$.

Let $v \in V_{s} \cap V_{\text {nil }}$. Then $v=F^{m}(w)$ for some $w \in V$. So, $0=F^{m}(v)=F^{2 m}(w)$ hence $w \in V_{\text {nil }}$. But this gives $F^{m}(w)=0$, or $v=0$. Therefore, $V_{s} \cap V_{\text {nil }}=0$.

Now, let $v \in V$. Since $F^{m}(v) \in V_{s}$ then $F^{m}(v)=F^{m+e_{0}}(w)$ for some $w \in V$. So, $F^{m}\left(v-F^{e_{0}}(w)\right)=0$ or in other words $v-F^{e_{0}}(w) \in V_{\text {nil }}$.

Now note that $v=v-F^{e_{0}}(w)+F^{e_{0}}(w)$ and that $F^{e_{0}}(w) \in F^{e_{0}}(V)=V_{s}$.

The following result has appeared first in [19], based on ideas of Lang, and then Hochster gave another proof more recently, see [17]. It extends Dieudonné's result, Theorem 6.6, in a complementary way to Theorem 6.7. 
Theorem 6.10. Let $V$ be a $K$-vector space and $K$ a separably closed field. Then $V_{S}$ admits a basis that is fixed by $F$.

\section{The Antinilpotent Case}

In this section we will present some applications to the case of antinilpotent vector spaces. The notion of an antinilpotent module was introduced in [13] with respect to modules that admit a Frobenius action. It has applications in the study of local cohomology modules of a local ring $A$ with support in its maximal ideal. These local cohomology modules are Artinian, but not finitely generated. However, to understand the notion of antinilpotency it is natural to first consider it in the context of simpler situations, such as finite dimensional vector spaces, and finitely generated modules over a commutative Noetherian ring. The treatment of vector spaces with Frobenius action which is the subject of the paper will be applied in this section.

We will first start with reviewing the antinilpotency of modules. In this section, $A$ denotes a commutative Noetherian ring of positive characteristic $p$ where $p$ is prime. Let $M$ be an $A$-module and assume that there exist an additive map $F=F_{M}: M \rightarrow$ $M$ such that $F(a m)=a^{p} F(m)$ for all $a \in A, m \in M$. We call $F=F_{M}$ a Frobenius action on $M$.

Definition 7.1. Let $M$ be an $A$-module that admits a Frobenius action $F: M \rightarrow M$. We say that $M$ is antinilpotent (with respect to $F$ ) if for any F-compatible (or Finvariant) submodule $N$ the induced map $F: M / N \rightarrow M / N$ is injective. This is equivalent to the condition that $F$ does not act nilpotently on (equivalently, does not kill) any nonzero subquotient $Q / N$ of F-compatible submodules $Q, N$ of $M$.

Let $S \subset A$ be a multiplicative set and $M$ be an $A$-module with a Frobenius action. Then $F$ extends naturally to a Frobenius action on $S^{-1} M$ by $F\left(\frac{m}{s}\right)=\frac{F(m)}{s^{p}}$ for all $m \in M, s \in S$. If $M$ is antinilpotent, then $S^{-1} M$ is antinilpotent as well.

The following result represents the motivation behind the study of antinilpotent modules. For a commutative ring $A$ of prime characteristic $p$, we say that $A$ is $\mathrm{F}$ pure if $F: A \rightarrow A$ is a pure homomorphism of $A$-modules.

Theorem 7.2 (Enescu-Hochster, [13]). Let $(A, \mathfrak{m})$ be an F-pure Gorenstein local ring of dimension d. Then $H_{\mathfrak{m}}^{d}(A)$ is antinilpotent with respect to the canonical Frobenius action on it.

Under the Gorenstein hypothesis, the F-purity of $A$ is equivalent to the injectivity of the Frobenius action on $H_{\mathfrak{m}}^{d}(A)$. So, when $A$ is Gorenstein, Theorem 7.2 says that the injectivity of the Frobenius action implies a stronger form of injectivity, namely antinilpotency. Therefore, it makes sense to understand the connection between these two concepts in a general setting.

An interesting property of antinilpotent modules is highlighted by the next result. 
Theorem 7.3 (Enescu-Hochster, [13]). Let $M$ be an Artinian A-module. Assume that $M$ admits a Frobenius action $F$ such that $M$ is antinilpotent. Then $M$ has finitely many submodules that are F-compatibile.

Let $M$ be an $A$-module and $F: M \rightarrow M$ a Frobenius action on $M$. For a submodule $N$ of $M$ we denote $\langle F(N)\rangle_{A}$ the $A$-submodule generated by $F(N)$ in $M$.

Lemma 7.4. Let $M$ be an A-module, and $F: M \rightarrow M$ an injective Frobenius action on $M$. Then $M$ is antinilpotent if and only if for all $F$-compatible submodules $N$ of $M,\langle F(N)\rangle_{A}=N$.

Proof. First assume that $M$ is antinilpotent and let $N$ be an F-compatible submodule of $M$. Then we have an induced Frobenius action $F: \frac{N}{\langle F(N)\rangle_{A}} \rightarrow \frac{N}{\langle F(N)\rangle_{A}}$ which is trivially kills $\frac{N}{\langle F(N)\rangle_{A}}$. Thefore, $N=\langle F(N)\rangle_{A}$, since $M$ is antinilpotent.

For the converse, let $N$ be an F-compatible submodule of $M$. We plan to show that $F: \frac{M}{N} \rightarrow \frac{M}{N}$ is injective, or, in other words, if $m \in M$ with $F(m) \in N$, then $m \in N$.

Let $N_{0}=R m+N$. Clearly, $F\left(N_{0}\right) \subset\left\langle F\left(N_{0}\right)\right\rangle_{A} \subseteq\langle F(N)\rangle_{A} \subseteq N \subseteq N_{0}$ which means that $N_{0}$ is F-compatible. By hypothesis, $\left\langle F\left(N_{0}\right)\right\rangle_{A}=N_{0}$, so $N=N_{0}$. Therefore $m \in N$.

Let $A$ be a ring and $\mathfrak{m}$ a maximal ideal of $A$. Let $V$ be a vector space over $A / \mathfrak{m}$ and consider the natural $A$-module structure on $V$ obtained by restriction of scalars to $A$, denoted by $V(\mathfrak{m})$. As sets $V=V(\mathfrak{m})$. Obviously, if $F$ is a Frobenius action on $V$ over $A / \mathfrak{m}$, one obtains a natural Frobenius action on $V(\mathfrak{m})$ as an $A$-module.

Proposition 7.5. (i) Let $(A, \mathfrak{m})$ be a local ring and $M$ a finitely generated $A$-module with an antinilpotent Frobenius action. Then $\mathfrak{m} M=0$ and hence $M=V(\mathfrak{m})$, where in fact $V=M$ regarded as $A / \mathrm{m}$-vector space and $V$ is finite-dimensional and antinilpotent.

(ii) Let $A$ be a Noetherian ring and $M$ be an antinilpotent module that is finitely generated over $A$. Then there exist a finite set $\Lambda$ of maximal ideals of $A$ and antinilpotent $A / \mathfrak{m}_{i}$-vector spaces $V_{i}$, for any $\mathfrak{m}_{i} \in \Lambda$ such that $M=$ $\bigoplus_{\mathfrak{m}_{i} \in \Lambda} V_{i}\left(\mathfrak{m}_{i}\right)$.

Proof. For (i), for any ideal $I, F$ acts injectively on $I M / I^{2} M$. On the other hand $F$ kills $I M / I^{2} M$ and so $I M=I^{2} M$. Therefore $I M=0$ and in particular $\mathfrak{m} M=0$. The rest of assertions follow.

(ii) Again $I^{2} M=I M$ for all $I$ ideals in $A$ and this is preserved under localization. Note that $M \rightarrow \prod_{\mathfrak{m} \in \operatorname{Max}(A)} M_{\mathfrak{m}}$ is injective. By (1) $\mathfrak{m} M_{\mathfrak{m}}=0$. So $M_{\mathfrak{m}}=V(\mathfrak{m})$ for some $A / \mathfrak{m}$-vector space $V$. Now it follows easily that the image of $M$ is of the form stated in the conclusion. The fact the only a finite number of maximal ideals appear in the product giving $M$ comes from the fact that $M$ is Noetherian. 
Let $F_{*} A=A^{(1)}$ be the right $A$-algebra obtained on $A$ via the Frobenius map $F$ : $A \rightarrow A$ by restriction of scalars. By convention, $A^{(1)}$ equals $A$ as a left $A$-module. Let $M$ be an $A$-module with a Frobenius action $F_{M}: M \rightarrow M$. Let $F_{*}(M)=$ $A^{(1)} \otimes_{A} M$.

It is easy to check that the Frobenius actions on $M$ are in one-to-one correspondence to elements of $\operatorname{Hom}_{A}\left(F_{*}(M), M\right)$ (as in Remark 3.2 in [25]). Under this correspondence $F_{M}$ maps to $\omega_{M}$, where $\omega_{M}(a \otimes m)=a F(m)$, for all $a \in A, m \in M$.

This construction is functorial in the sense that if $g: N \rightarrow M$ is an $A$-linear map with compatible Frobenius action

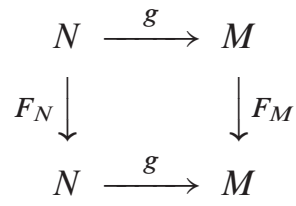

then the following diagram of $A$-linear maps

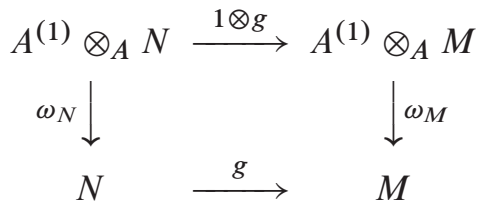

commutes.

An example of an $A$-linear map compatible with the Frobenius actions is the inclusion map $i: N \rightarrow M$ defined by an F-compatible submodule $N$ of $M$. Note that $\langle F(N)\rangle_{A}=\omega_{N}\left(A^{(1)} \otimes_{A} N\right)$.

Proposition 7.6. Let $V$ be a finite dimensional vector space with a Frobenius action $F: V \rightarrow V$ and corresponding $\omega_{V}: K^{(1)} \otimes_{K} V \rightarrow V$. Then $V$ is antinilpotent if and only if $\omega_{V}$ is isomorphism if and only if $\omega_{V}$ is injective if and only if $\omega_{V}$ is surjective.

Proof. The composition of $V \rightarrow K^{(1)} \otimes_{K} V \stackrel{\omega_{V}}{\rightarrow} V$ gives the Frobenius action on $V$ so the injectivity of $\omega_{V}$ implies that $F$ is injective.

First note that $\operatorname{dim}_{K^{(1)}}\left(K^{(1)} \otimes_{K} V\right)=\operatorname{dim}_{K}(V)$. But on the left $K^{(1)}$ is simply $K$. Therefore the surjectivity of $\omega_{V}$ implies that $\omega_{V}$ is an isomorphism of $K$-vector spaces.

Now consider an F-compatible $K$-subspace $V^{\prime}$ of $V$. Since $K$ is field we get that $K^{(1)} \otimes_{K} V^{\prime} \stackrel{i}{\hookrightarrow} K^{(1)} \otimes_{K} V$. Moreover we have the following commutative diagram

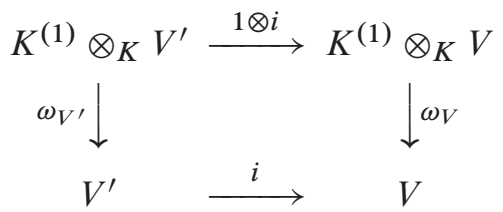


which implies that $K^{(1)} \otimes_{K} V^{\prime} \rightarrow V^{\prime}$ is injective. As above, by dimension considerations, it must also be surjective, hence $\left\langle F\left(V^{\prime}\right)\right\rangle_{K}=V^{\prime}$ which implies that $V$ is antinilpotent, according to Lemma 7.4.

For the converse, if $V$ is antinilpotent then $\omega_{V}\left(K^{(1)} \otimes_{K} V\right)=V$ by Lemma 7.4, hence $\omega_{V}$ is surjective

Corollary 7.7. Let $K$ be a perfect field. Let $V$ be a finite dimensional $K$-vector space that admits a Frobenius action. Then the Frobenius action is injective if and only if $V$ is antinilpotent.

Proof. Fix a basis for $V$. If the Frobenius action $F$ is injective, then Proposition 6.4 shows that the matrix describing the action is invertible. But this matrix coincides with the one describing the map $\omega_{V}$ therefore Theorem 7.6 applies.

Corollary 7.8. Let $K \subseteq L$ be a finite field extension. Consider the Frobenius map on $L$ which can be regarded as a Frobenius action of $L$ as $K$-vector space.

Then $L$ is antinilpotent over $K$ if and only if $K \subset L$ is separable.

Proof. It suffices to note that $K^{(1)} \otimes_{K} L \rightarrow L$ is injective if and only if $K \subset L$ is separable.

We would like to give now an example of a finite dimensional $K$-vector space $V$ that admits an injective Frobenius action, but it is not antinilpotent. Note that the base field $K$ is not perfect.

Example 7.9. Let $\alpha$ be a solution of the equation $t^{3}+t^{2}=1$ over $\mathbb{F}_{3}$. Let $X$ be an indeterminate and construct $K=\mathbb{F}_{3}(\alpha)(X) \subset L=\mathbb{F}_{3}(\alpha)(\sqrt[3]{X})$.

Let $V=L^{2}$. Since $L$ is finite over $K$ of degree 3 then $\operatorname{dim}_{K}(V)=6$.

We define the following Frobenius action

$$
F\left(x_{1}, x_{2}\right)=\left(x_{1}^{3}, x_{2}^{3}\right) \cdot\left(\begin{array}{ll}
1 & 1 \\
0 & 1
\end{array}\right)=\left(x_{1}^{3}, x_{1}^{3}+x_{2}^{3}\right),
$$

for all $x_{1}, x_{2} \in L$.

It is an immediate check to see that $F$ is injective.

Let $W=K \cdot(\alpha,-1) \subset V$.

Let $a \in K$. Then $F(a(\alpha,-1))=a^{3} F(\alpha,-1)=a^{3}\left(\alpha^{3}, \alpha^{3}-1\right)=a^{3}\left(\alpha^{3},-\alpha^{2}\right)=$ $\alpha^{2} \cdot a(\alpha,-1) \notin W$, since $\alpha^{2} \notin \mathbb{F}_{3}$.

But if $v=\sqrt[3]{X}(\alpha,-1)$, we get $F(v)=\alpha^{2} \cdot X(\alpha,-1) \in W$, while $v \notin W$.

This shows that $V$ is not antinilpotent with respect to the action considered.

We explain now a relationship between the notion of $\mathbb{F}$-modules, due to Lyubeznik [23] who in turn was inspired by work of Harthsorne-Speiser [16], and antinilpotent modules. 
Definition 7.10. Let $M$ be an $A$-module with a Frobenius action $F: M \rightarrow M$. We say that $M$ is an $\mathbb{F}$-module if the map

$$
A^{(1)} \otimes_{A} M \rightarrow M
$$

is an $A$-isomorphism of modules. Note that, as an left $A$-algebra, $A^{(1)}=A$.

Over a regular ring $A$, every $\mathbb{F}$-module $M$ has the property that a submodule $N$ is an $\mathbb{F}$-module if and only if $\langle F(N)\rangle_{A}=N$.

Corollary 7.11. A finite dimensional vector space $V$ over a field $K$ is an $\mathbb{F}$-module if and only if it is antinilpotent.

When $K=\mathbb{F}_{p}$ we note that a Frobenius action of a $K$-vector space is simply a $K$ linear function. In this situation the antinilpotency of $V$ is equivalent to the injectivity of $F$ on $V$ as it is clear from the characterization in Theorem 7.6. In this case since $V$ is finite is clear that there are finitely many (F-compatible) subspaces of $V$.

One question that arises in this context is the following. Assume that $K$ is now an (infinite) field and let $T: V \rightarrow V$ be a $K$-linear map. Is it true that there are at most finitely many subspaces $V^{\prime}$ of $V$ compatible with $T$, i.e. $T\left(V^{\prime}\right) \subset V^{\prime}$ ?

The reader can check that this is the case if and only if $V$ has no repeated elementary divisors, when $K$ is infinite.

We would like to close with an extension of Theorem 4.21 in [13] that shows how the results in this survey might be used in current research.

Proposition 7.12. Let $(A, \mathfrak{m})$ be a complete local ring with perfect residue field $K$. Assume that $A$ has prime characteristic $p$, and let $M$ be an A-module of finite length that admits a Frobenius action $F$. If $F$ acts injectively on $M$ then $M$ is antinilpotent with respect to $F$.

Proof. Note that $A$ contains a copy of $K$. Hence, since $M$ is of finite length over $A, M$ is a finite dimensional vector space over $K$. The action $F$ is injective on $M$ when $M$ is regarded as a $K$-vector space so by Corollary 7.7 it follows that $M$ is antinilpotent as a $K$-vector space. This automatically makes $M$ antinilpotent as an $A$-module as well.

Definition 7.13. Let $(A, \mathfrak{m})$ be a local ring of dimension $d$. We say that $A$ is generalized Cohen-Macaulay if $H_{\mathfrak{m}}^{i}(A)$ is of finite length over $A$, for all $i<d$. A local ring of prime characteristic $p>0(A, \mathfrak{m})$ is called $\mathrm{FH}$-finite if, for all $i=0, \ldots, d$, the module $H_{\mathfrak{m}}^{i}(A)$ has only finitely many F-compatible submodules.

Theorem 7.14. Let $(A, \mathfrak{m})$ be a local, complete domain. Assume that $A$ is F-injective, generalized Cohen-Macaulay with perfect residue field $K$. If the test ideal of $A$ is m-primary, then $\mathrm{A}$ is $\mathrm{FH}$-finite. 
Proof. Since for all $0 \leq i \leq d-1, H_{\mathfrak{m}}^{i}(A)$ are of finite length over $A$, we have that each $H_{\mathfrak{m}}^{i}(A)$ is antinilpotent with regard to the natural Frobenius action because this action is injective, by Proposition 7.12.

Let $V=0_{H}^{*}$ where $H=H_{\mathfrak{m}}^{d}(A)$. Denote by $\tau$ the test ideal of $A$. Then it is known that $\tau \cdot V=0$ and that $V$ is the largest proper F-compatible submodule of $H$ by a theorem of K. E. Smith. Since $V$ is Artinian and $\tau$ is m-primary it follows that $V$ is of finite length over $A$. Again we conclude that $V$ is antinilpotent, by Proposition 7.12.

To conclude that the action on $H$ is antinilpotent we need to show that $F$ acts injectively on the quotient $H / V$. But $H / V$ is a simple $K\{F\}$-module. This means that we only need to check that $F$ does not kill $H / V$.

Let $x_{1}, \ldots, x_{d}$ a system of parameters for $A$. Think of $H$ as the last cohomology module in Čech complex associated to $x_{1}, \ldots, x_{d}$. The elements of $H$ can be thought of as classes $\left[\frac{a}{\left(x_{1} \cdots x_{d}\right)^{t}}\right]$ with $a \in A$ and $t \geq 0$. Denote by 1 the element $\left[\frac{1}{x_{1} \cdots x_{d}}\right]$. Note that this element generates $H$ as an $A\{F\}$-module. Now assume that $F$ kills $H / V$. Then $F(1) \in V$. But $F(1)$ also generates $H$ as an $A\{F\}$-module. So this implies that $H \subseteq V$ which is a contradiction.

Therefore $H$ and hence all modules $H_{\mathfrak{m}}^{i}(A)$ are antinilpotent with respect to the canonical Frobenius action. But then Theorem 4.14 in [13] shows that $A$ is FH-finite.

Acknowledgments. I thank Lee Klingler for informing me of the work of Jacobson in [26], Manuel Blickle and the referee for corrections, and Yongwei Yao for corrections and suggestions that helped to improve the paper.

\section{Bibliography}

[1] Asano, K., Nichtkommutative Hauptidealringe, vol. 696 of Actualités. sci. indust. Hermann, Paris 1938.

[2] Asano, K., Arithmetische Idealtheorie in nichtkommutativen Ringen. Jap J Math. 1939; 16:1-36.

[3] Aberbach, I. M., Enescu, F., Test ideals and base change problems in tight closure theory. Trans Amer Math Soc. 2003;355(2):619-636 [electronic].

[4] Blickle, M., The intersection homology $\mathscr{D}$-module in finite characteristic [PhD thesis]. University of Michigan; 2001.

[5] Blickle, M., The intersection homology $\mathscr{D}$-module in finite characteristic. Math Ann. 2004;328(3):425-450.

[6] Blickle, M., Intersection homology $\mathscr{D}$-modules in finite characteristic. In: Mathematisches Institut, Georg-August-Universität Göttingen: Seminars 2003/2004. Göttingen: Universitätsdrucke Göttingen; 2004. p. 91-98.

[7] Blickle, M., Singularities in positive characteristic via D-modules and Frobenius [habilitation thesis]. Universität Essen; 2007. 
[8] Blickle, M., Minimal $\gamma$-sheaves. Algebra Number Theory. 2008;2(3):347-368.

[9] Blickle, M., Böckle, G., Cartier modules: finiteness results. arXiv:math/0909.2531.

[10] Dieudonné, J., Lie groups and Lie hyperalgebras over a field of characteristic $p>0$ II. Amer J Math. 1955;77:218-244.

[11] Enescu, F. $F$-injective rings and $F$-stable primes. Proc Amer Math Soc. 2003;131(11): 3379-3386.

[12] Enescu, F., Local cohomology and F-stability. J Algebra. 2009;322(9):3063-3077.

[13] Enescu, F., Hochster, M., Frobenius structure of local cohomology. Algebra Number Theory. 2008;2(7):721-754.

[14] Fedder, R., Watanabe, K.-I., A characterization of $F$-regularity in term of $F$-purity. In: Commutative algebra. vol. 15 of Mathematical Sciences Research Institute publications. 1989. p. 227-245.

[15] Goodearl, K. R., Warfield Jr, R. B., An introduction to noncommutative Noetherian rings. vol. 16 of London Mathematical Society student texts. Cambridge University Press; 1989.

[16] Harthsorne, R., Speiser, E., Local cohomological dimension in characteristic $p>0$. Ann Math. 1977;105:45-79.

[17] Hochster, M., Some finiteness properties of Lyubeznik's $\mathcal{F}$-modules. In: Algebra, geometry and their interactions. vol. 448 of Contemporary Mathematics. Providence, RI: American Mathematical Society; 2007. p. 119-127.

[18] Hochster, M., Roberts, J. L., The purity of the Frobenius and local cohomology. Adv Math. 1976;21(2):117-172.

[19] Katz, N., Une formule de congruence pour la fonction $\xi$, Expose XXII. In: Deligne $\mathrm{P}$, Katz N, editors. Groupes de monodromie en géométrie algébrique. II, Séminaire de Géométrie Algébrique du Bois-Marie 1967-1969 (SGA 7 II). vol. 340 of Lecture notes in mathematics. Berlin-New York: Springer-Verlag; 1973.

[20] Katzman, M., Parameter-test-ideals of Cohen-Macaulay rings. Compos Math. 2008; 144(4):933-948.

[21] Katzman, M., A non-finitely generated algebra of Frobenius maps. Proc Amer Math Soc. 2010;138(7):2381-2383.

[22] Katzman, M., Frobenius maps on injective hulls and their applications to tight closure. J Lond Math Soc (2). 2010;81(3):589-607.

[23] Lyubeznik, G., $F$-modules: applications to local cohomology and $D$-modules in characteristic $p>0$. J Reine Angew Math. 1997;491:65-130.

[24] Lyubeznik, G., On the vanishing of local cohomology in characteristic $p>0$. Compos Math. 2006;142(1):207-221.

[25] Lyubeznik, G., Smith, K. E., On the commutation of the test ideal with localization and completion. Trans Amer Math Soc. 353:8 (2001); 3149-3180.

[26] Jacobson, N., The theory of rings. vol. 2 of Mathematical surveys and monographs. New York: American Mathematical Society; 1943. 
[27] Jacobson, N., Basic Algebra II. 2nd ed. Dover Publications; 2009.

[28] McConnell, J. C., Robson, J. C., Noncommutative Noetherian rings [with the cooperation of Small, L. W.]. Revised ed. vol. 30 of Graduate studies in mathematics. Providence, RI: American Mathematical Society; 2001.

[29] Nakayama, T., A note on the elementary divisor theory in non-commutative domains. Bull Amer Math Soc. 1938;44(10):719-723.

[30] Schwede, K., Centers of F-purity. Math Z. 2010;265(3):687-714.

[31] Schwede, K., Tucker, K., On the number of compatibly Frobenius split subvarieties, prime $F$-ideals, and log canonical centers. Ann Inst Fourier (Grenoble). 2010;60(5): $1515-1531$.

[32] Sharp, R. Y., On the Hartshorne-Speiser-Lyubeznik theorem about Artinian modules with a Frobenius action. Proc Amer Math Soc. 2007;135(3):665-670 [electronic].

[33] Sharp, R. Y., Graded annihilators of modules over the Frobenius skew polynomial ring, and tight closure. Trans Amer Math Soc. 2007;359(9):4237-4258 [electronic].

[34] Sharp, R. Y., Graded annihilators and tight closure test ideals. J Algebra. 2009;322(9): 3410-3426.

[35] Sharp, R. Y., An excellent $F$-pure ring of prime characteristic has a big tight closure test element. Trans Amer Math Soc. 2010;362(10):5455-5481.

[36] Sharp, R. Y., Yoshino, Y., Right and left modules over the Frobenius skew polynomial ring in the F-finite case. Math Proc Cambridge Philos Soc. To appear.

[37] Smith, K. E., Tight closure of parameter ideals. Invent Math. 1994;115(1):41-60.

[38] Smith, K. E., Test ideals in local rings. Trans Amer Math Soc. 1995;347(9):3453-3472.

[39] Smith, K. E., The $D$-module structure of $F$-split rings. Math Res Lett. 1995;2(4):377386.

[40] Smith, K. E., F-rational rings have rational singularities. Amer J Math. 1997;119(1): 159-180.

[41] Smith, K. E., Vanishing, singularities and effective bounds via prime characteristic local algebra. Proceedings, Algebraic Geometry; 1995, Santa Cruz. vol. 62, part 1 of Proceedings of symposia in pure mathematics. Providence, RI: American Mathematical Society; 1997. p. 289-325.

[42] Teichmüller, O., Der Elementarteilersatz für nichtkommutative Ringe. Sitzungsberichte der Preussischen Akademie der Wissenschaften. 1937.

[43] Yoshino, Y., Skew-polynomial rings of Frobenius type and the theory of tight closure. Comm Algebra. 1994;22(7):2473-2502.

\section{Author Information}

Florian Enescu, Department of Mathematics and Statistics, Georgia State University, Atlanta GA, USA.

E-mail: fenescu@gsu.edu 